\title{
Estany, A. y Puyol, Á. (eds.) (2016). Filosofía de la epidemiología social. Plaza y Valdés-CSIC. Colección Theoria cum Praxi. 268 pp.
}

El título de este libro nos invita a reflexionar sobre la siguiente pregunta: ¿qué distingue a la epidemiología social de la propia epidemiología? A primera vista parecería extraña y redundante una epidemiología que no fuera social, en cuanto el objeto de estudio de la epidemiología es la salud y enfermedad de poblaciones. Con el fin de distinguir a la epidemiología social de la propia epidemiología, veamos antes algo de su historia.

Es bien sabido que la epidemiología surge como disciplina de estudio en la Inglaterra decimonónica con el descubrimiento de John Snow del modo de transmisión del cólera (Vinten-Johansen et al., 2003). Como nos recuerda Segura en su capítulo Breves notas contextuales acerca (del desarrollo) de la epidemiología, la epidemiología surge cuando la concepción miasmática de la enfermedad se sustituye por la noción de infección por agentes patógenos (cfr. pp. 48-49), y el caso de Snow es paradigmático de lo que hoy consideramos el contagio de enfermedades infecciosas. Más recientemente, cuando otro tipo de agentes (como la nicotina y otros químicos del tabaco) son reconocidos como factores de riesgo de ciertas enfermedades (cáncer de pulmón), se comienza a hablar de factores ambientales y es cuando se consideran factores de riesgo estrictamente sociales, como lo muestran Estrach y Vallerdú en su texto Epidemiología de las enfermedades mentales: ¿un caso aparte? con respecto a la relación entre condiciones laborales precarias y la depresión. Es por estos casos de determinantes sociales que, para muchos estudiosos, la epidemiología social tiene lugar como una disciplina autónoma de investigación.

Pero la epidemiología social tiene antecedentes más antiguos en el siglo XIX con las observaciones de Villermé (1782-1863) y Virchow (18211902), quienes refinaron sus hallazgos incluyendo condiciones sociales y económicas como determinantes de la enfermedad. Villermé afirmó que el avance de la civilización era un factor importante para explicar las epidemias, como en el caso del cólera, que afectaba más a los pobres y en ciudades menos desarrolladas (Villermé, 1976). Por el otro lado, en su reporte de 1848 sobre el tifus en el norte de Silesia registró y destacó las condiciones de hábitat, higiene, dieta y salubridad como las principales causas de la enfermedad (Taylor y Rieger, 1984). En ese mismo siglo, adherentes a la teoría miasmática de la enfermedad como William Farr 
y Edwin Chadwick enfatizaban la relación entre pobreza y enfermedad. Sin embargo, la epidemiología continuó interesándose principalmente por los determinantes biológicos o ambientales de la salud. Fue hasta las décadas de los sesenta y setenta del siglo XX cuando epidemiólogos como John Cassels, Mervyn Susser, S. Leonard y otros desarrollaron una epidemiologia centrada en el impacto que tienen las condiciones sociales en la salud. Así, si bien tanto la epidemiología general como la epidemiología social tienen sus antecedentes en investigaciones del siglo XIX, la primera se constituyó como tal mostrando estudios sistemáticos desde ese periodo, mientras que la segunda vio su conformación teórica y práctica apenas a mediados del siglo XX.

Los primeros estudios representativos de la epidemiología social son los estudios White Hall I y II, realizados en Inglaterra en los años setenta del siglo XX (Honjo, 2004). Dichos estudios analizaron la relación entre las tasas de prevalencia de enfermedades cardiovasculares y las condiciones sociales. Esto lo hicieron mediante un estudio de cohorte aplicado a servidores públicos. El equipo que realizó los estudios encontró una fuerte conexión entre las tasas de incidencia y mortalidad por enfermedades cardiovasculares, por un lado, y las tasas de desempleo y bajo ingreso de los servidores públicos, por el otro. Asimismo, los resultados arrojaron una relación entre estos mismos factores (desempleo y bajo ingreso) y otros factores de riesgo, como lo son el hábito de fumar, una mala dieta y falta de ejercicio (Krieger, 2001 y Van Rossum et al., 2000).

En la actualidad, algunos de los estudios más importantes son los que analizan los determinantes sociales de la obesidad, como lo son aquellos que la relacionan con el espacio urbano y la inseguridad en una ciudad (Cutts et al., 1982). Dichos estudios se distinguen de la epidemiología general, enfocada actualmente en encontrar causas genéticas, en las que observan las interacciones y condiciones sociales de una población para poder entender la distribución de los estados de salud de ésta.

En nuestra región latinoamericana, la epidemiología social ha sido también una herramienta muy útil para estudiar la obesidad, como lo muestran investigaciones realizadas sobre el entorno urbano con respecto al acceso a alimentos, distinguiendo entre los que son altos en grasas saturadas y aquéllos que no lo son (Dávila-Torres et al., 2015). Asimismo, otros investigadores (Rodríguez-Torres y Casas-Patiño, 2018) identifican como determinantes sociales de la obesidad un salario mínimo insuficiente, la elevación del costo de la canasta básica y las 
condiciones laborales. Finalmente, en Figueroa (2009) se consideran como explicaciones de la epidemia de obesidad en Latinoamérica cambios relevantes en los patrones de alimentación, en la reducción de la actividad física y en otros aspectos socioculturales.

Pasemos ahora a la descripción del libro sobre el cual trata esta reseña. En este libro se convoca a filósofos de la ciencia, la ética y la política, a epidemiólogos y a expertos en políticas sanitarias con el fin de abordar diversos aspectos filosóficos que atañen a la epidemiología social. Los editores han organizado la presentación de doce artículos en tres grandes rubros: el análisis de la epidemiología social como campo disciplinar, su pertinencia filosófica de cara a la epistemología y la ética principalmente, así como su estrecha relación con áreas de la esfera pública, como lo son el derecho y la política.

Con respecto al primer tópico, los autores de este libro van más allá de la discusión sobre la pertinencia de la epidemiología social. Por un lado, como bien lo indica Estany en su capítulo Epidemiología ¿social? hacia un enfoque interdisciplinar, la epidemiología social, al centrar su objeto de estudio en los determinantes sociales, pone en entredicho la forma en que se dividen los saberes, particularmente la relación entre las ciencias biológicas y las sociales. Por otro lado, Porta, en su texto Si la epidemiología social existe, es integrativa. Y lo que importa es su influencia, teórica y práctica, aboga por la tesis de la integración, según la cual "la mejor teoría y la mejor praxis de la epidemiología social son aquellas que integran conocimientos, técnicas y métodos sobre los factores, mecanismos y procesos políticos, filosóficos, culturales, económicos, biológicos, físico-ambientales..." (p. 31).

Con respecto al segundo tópico del libro, los ensayos que componen este rubro abordan problemas epistemológicos de conceptos clásicos de la filosofía de la ciencia y claves para la epidemiología (como lo es la causalidad), retos y problemas éticos con respecto a los datos de los estudios epidemiológicos, así como problemas relacionados con la noción misma de enfermedad. En primer lugar, Jordi Vallerdú, en su texto Causalidad y epidemiología, muestra cómo han cambiado las teorías causales en esta disciplina desde el modelo monocausal dominante a fines del siglo XIX hasta la eco-epidemiología del siglo XXI, donde los determinantes sociales de la salud cobran una importancia que no tenían previamente. Los problemas estudiados son cada vez más complejos; se puede apreciar una miríada de datos y los factores se conciben relacionados estrechamente en sistemas complejos, lo cual ha 
implicado un cambio terminológico y metodológico para enfrentar los retos de la epidemiología en el siglo XXI. Muy ligado a este problema es el de la multifactorialidad: los estados de salud se deben a una multitud de factores. Como apunta David Casacuberta en su texto Prolegómenos a toda epidemiología futura que quiera presentarse como ciencia, los factores causales de un estado de salud interactúan entre sí de forma no lineal y se organizan de forma compleja. Esto trae consigo varios retos epistémicos y éticos, como lo es el problema de la distinción entre los factores causales y los de confusión. El ejemplo que muestra Casacuberta es la relación entre el cáncer y el café; algunos estudios sugerían una relación causal entre ellos cuando se trata más bien de un factor de confusión, ya que muchos fumadores son también adictos al café.

En segundo lugar, con respecto al manejo de los datos, la emergencia de las tecnologías digitales y el big data trae consigo tanto nuevos problemas como oportunidades. Por un lado, el exceso de información personal en la red requiere de controles más rigurosos para que ésta sea fiable a la vez que anónima, lo cual presenta retos éticos para la epidemiología de cara a cómo se maneja y se cuida dicha información. Por otro lado, como comenta el propio Casacuberta, la información obtenida de voluntarios podría ser más fiable y podría haber un mejor seguimiento. Estrach y Vallerdú, en su texto ya mencionado sobre enfermedades mentales, abogan por una epidemiología social basada en sistemas complejos como una solución a problemas de este tipo, así como al de la multifactorialidad causal en epidemiología. Como lo muestran estos autores, a veces los factores sociales son los que influyen en lo biológico.

En tercer lugar, queda al descubierto un problema epistémico con respecto a la definición de nuevas entidades mórbidas. Borrell y Estany, en su texto Construcción y refutación de nuevas enfermedades. El debate entre esencialistas y pragmáticos desde la perspectiva de la epidemiología social, ilustran muy bien el debate entre esencialistas y pragmáticos. Aun cuando la existencia de nuevas entidades, como lo es la fibromialgia, ha sido puesta en duda por muchos, su postulación cumple tanto una función social: "proporciona un diagnóstico al paciente, casi siempre con repercusiones morales y jurídicas" (p. 124), como una de agrupador epidemiológico: "permite agrupar a los pacientes con fines de observación y ensayos clínicos" (p. 124).

Con respecto al tercer tópico del libro, los ensayos que componen este rubro muestran cómo algunos problemas éticos y políticos se 
desprenden del conocimiento científico que genera la epidemiología social. En primer lugar, Andreu Ballús y Éric Arnau, en su texto La autonomía relativa de la epidemiología social con respecto a la ética, se preguntan si existe independencia entre las consideraciones éticas en epidemiología social y la investigación en la misma disciplina. Los autores argumentan que, dado que la epidemiología es una ciencia de tipo cluster, orientada a objetivos prácticos y no necesariamente a la generación de conocimientos, es imposible separar las cuestiones éticas de la propia disciplina. Sin embargo, argumentan que los sistemas éticos son independientes de la metodología y la generación de datos y que los valores elegidos en una investigación deben hacerse explícitos. Puyol, en su texto Ética y epidemiología social, argumenta que los determinantes sociales de una cierta población inducen desigualdades en virtud de que son dependientes de factores sociales más que de hábitos voluntarios y de la propia biología. La epidemiología, en su relación con la política y el derecho, busca eliminar desigualdades mediante políticas públicas y legislaciones. Esto significa, de acuerdo con Puyol, que la epidemiología tiene una dimensión ética que es relevante en la aplicación de sus conocimientos. Por otra parte, Gamper, en su texto Epidemiología y ley: de la cuarentena al litigio de la responsabilidad civil, alude a dos aspectos de la relación con el derecho: por un lado, el uso - a veces coercitivode la ley para la vigilancia y cuidado de la salud pública, $\mathrm{y}$, por otro lado, los juicios de responsabilidad civil. En estos dos aspectos están en constante tensión dos bienes fundamentales de toda sociedad, el público y el individual. Un ejemplo del primero son los programas de vacunación, los cuales, si bien buscan tutelar la salud y controlar posibles epidemias mediante programas inmunológicos, reconocen la objeción de conciencia, según la cual cualquier ciudadano puede desacatar la obligación de vacunarse apelando a razones religiosas, por ejemplo. El segundo aspecto resalta el papel de las pruebas epidemiológicas como evidencia en casos de litigio de responsabilidad civil, como la exposición a asbesto y su relación con el cáncer de pulmón, por ejemplo. La gran dificultad en este caso es la tensión entre la verdad científica, que usualmente postula una causa en términos probabilísticos, y la culpabilidad jurídica, que pretende conseguir pruebas suficientes para probar la culpabilidad que se reclama.

Así, la unión entre epidemiología y Estado para intervenir sobre los ciudadanos invita a algunos a estudiar los aspectos ideológicos y políticos de esta unión, como es el caso de Farrés Juste, quien, en su texto 
Ideología y epidemiología, a partir de ideas de Hegel y de Kojève argumenta que el Estado universal y homogéneo es el sustento ideológico de la epidemiología, perspectiva desde la cual lo privado se vuelve público y objeto de intervención. Todo esto apunta a una relación estrecha entre el conocimiento epidemiológico y el poder ejercido por el Estado. En esta línea, Quintanas, en su texto Epidemiología y biopolítica, introduce el concepto foucaultiano de biopolítica y lo aplica al estudio de las relaciones de poder construidas desde la epidemiología social. La autora muestra cómo históricamente la salud pública se ha convertido en un instrumento de vigilancia y control sobre los cuerpos de los individuos y cómo - entre otras cosas - esto contribuye a la medicalización de la sociedad. Por lo tanto, es necesario estudiar las relaciones de poder relevantes al mismo tiempo que los estados de salud de la población.

En resumen, este libro ofrece, por una parte, un estudio sintético y erudito de la historia de la epidemiología - cubriendo incluso episodios de la propia España-, un análisis filosófico de las distintas nociones de causalidad que la han permeado, así como, por otra parte, una reflexión metodológica sobre el análisis de datos en la era del big data. Este libro ofrece una perspectiva muy crítica y completa de los retos tanto epistémicos como éticos, jurídicos y políticos que enfrenta la epidemiología social actualmente.

Antes de concluir, pasamos a ofrecer una breve reflexión sobre el impacto de la investigación vertida en este libro en los estudios de Ciencia, Tecnología y Sociedad (CTS), así como una comparación con investigaciones existentes en nuestra región latinoamericana. Sobre lo primero: los avances científicos y tecnológicos propios de nuestra época han permeado a la epidemiología social tanto en su materia de estudio como en su metodología. Progresos tecnológicos en la producción y acceso a alimentos repercuten directamente en las condiciones de salud relacionadas con la obesidad, por ejemplo. Asimismo, el estudio de las desigualdades sociales inducidas por los determinantes sociales es otro tema en el corazón de los estudios CTS y uno de los principales en algunos ensayos de este libro. Con respecto a la metodología, como bien se apunta en este libro, la emergencia de las tecnologías digitales y el big data abre posibilidades para analizar la información de una manera mucho más extensa y transversal que como se había hecho hasta ahora con otros métodos. Pero estas posibilidades traen consigo retos éticos y jurídicos en el manejo de los datos confidenciales de las personas. Así, el desarrollo de la epidemiología social no puede entenderse sin una 
reflexión interdisciplinar, propia de los estudios CTS. De hecho, este libro es una muestra representativa y actual de la investigación sobre la filosofía de la salud pública llevada a cabo por miembros e interlocutores del Grupo de Estudios Humanísticos sobre la Ciencia y la Tecnología (GEHUCT), con sede en la Universidad Autónoma de Barcelona.

Sobre el lugar que ocupa este libro con respecto a la investigación existente en nuestra región, podemos decir que, si bien en Latinoamérica no ha primado una reflexión filosófica sobre la epidemiología como la realizada por los autores del libro reseñado, desde luego que hay reflexiones en torno a la epidemiología social. Un enfoque digno de mención es el que se materializa en la epidemiología sociocultural, también llamada epidemiología crítica (Cabellos y Quitral, 2007; HerschMartinez, 2013; Menéndez, 2008). Este enfoque enfatiza los aspectos sociales y culturales que influyen en la salud de la población, y es, en este sentido, muy similar a la epidemiología social. Sin embargo, existen diferencias sustantivas. Por un lado, la epidemiología social resulta en una metodología muy clara sobre el tipo de determinantes sociales que se estudian. Su principal base teórica podría considerarse el modelo ecosocial propuesto por Mervyn Susser (Susser y Stein, 2009; Susser, 1987), en el que los estados de salud son analizados a partir de diferentes niveles: genético, conductual y social, y donde los tres niveles interactúan entre sí. Por otro lado, la epidemiología sociocultural amplía el campo de la epidemiología social al considerar el tipo de intervenciones a realizar para mejorar el estado de salud de la población. A partir del concepto de daño evitable, la epidemiología sociocultural busca hacer explícitas las cuestiones sanitarias con el fin de intervenir social y culturalmente hacia la prevención de enfermedades. Se consideran dentro del campo de estudio las injusticias sociales, económicas, laborales y de género, así como temas relacionados con las poblaciones indígenas (HerschMartinez, 2013).

De igual modo, la epidemiología sociocultural es multicultural en la medida en que considera tanto la cultura del epidemiólogo como la de la población estudiada. Ésta es quizás la mayor aportación de esta nueva forma de hacer epidemiología. La consideración de los aspectos culturales obliga a pensar en una epidemiología que reconozca la cultura de la población y que busque conocimiento e intervenciones con ella y desde ella. De un modo similar, en Menéndez (2008) se entiende a la epidemiología sociocultural como un cruce entre la epidemiología y la antropología médica, tomando métodos y consideraciones teóricas 
de ambas, pero constituida como una disciplina aparte. Finalmente, la epidemiología sociocultural afirma que tanto la forma de entender la enfermedad como las respuestas ante aquélla están determinadas por el conjunto de prácticas simbólicas del grupo cultural en el que existen (Cabellos y Quitral, 2007). Así, aquella propuesta resulta ser tanto un buen complemento como una comparación pertinente con el enfoque de la epidemiología social del presente libro. Es un buen complemento ya que promueve una reflexión sobre la epidemiología social desde la propia epidemiología; es una comparación pertinente ya que permite ver las diferencias y similitudes entre diversas preocupaciones teóricas sobre los aspectos sociales y culturales de la epidemiología en distintas regiones del planeta.

El análisis filosófico de los aspectos metodológicos, epistémicos, éticos, jurídicos y políticos que enfrenta la epidemiología social del siglo XXI, presentada en los ensayos que componen este libro, contribuye de manera considerable a la discusión actual, particularmente en los países de habla hispana, en donde no existe a la fecha otro texto sobre la filosofía de la epidemiología social. Recomendamos ampliamente su lectura.

A modo de conclusión, terminamos con una reflexión sobre la reciente pandemia causada por el SARS-CoV-2. Hoy día es claro que las pandemias por enfermedades infecciosas no se han erradicado, como inocentemente algunos creían. Ante esta situación, la epidemiología social tiene un papel importante que jugar al identificar los determinantes sociales de los estados de salud de las poblaciones afectadas, así como al recomendar intervenciones para mitigar la pandemia. Un ejemplo de esto ha sido un estudio sobre el índice de vulnerabilidad publicado por la Universidad Nacional Autónoma de México. ${ }^{1}$ Entre otras cosas, dicha investigación hace explícita la relación que hay entre el número de muertes y determinantes sociales como el acceso a los servicios de salud. El análisis filosófico de dichos problemas es de gran importancia en este tipo de situaciones, ya que pretende responder a preguntas como las siguientes: ¿Cómo debe entenderse la causa de una epidemia?, ¿cuáles son los límites de intervención del Estado durante una epidemia?, ¿cómo debe usarse la tecnología? Éstas son preguntas que de una u otra forma

1 Puede consultarse en https://www.gits.igg.unam.mx/iCOVID-19/home. 
el libro aquí reseñado analiza. En estos tiempos de incertidumbre, la filosofía puede ayudar a brindar un poco de claridad.

\section{Bibliografía}

Cabellos, F. J. y Quitral, J. C. (2007). Epidemiología "clásica", epidemiología sociocultural y etnoepidemiología. Bases para una epidemiología intercultural en el desarrollo de modelos interculturales de salud en la región de La Araucanía. Actas del $6^{0}$ Congreso Chileno de Antropología. Tomo I. (pp. 134-149). Colegio de Antropólogos de Chile. URL: https://www.aacademica.org/ vi.congreso.chileno.de.antropologia/13.

Cutts, B., Darby, K., Christopher, B. y Brewis, A. (1982). City Structure, Obesity and Environmental Justice: An Integrated Analysis of Physical and Social Barriers to Walkable Streets and Park Access. Social Science y Medicine, 69, 1314-1322.

Dávila-Torres, J., González-Izquierdo, J. y Barrera-Cruz, A. (2015). Panorama de la obesidad en México. Revista médica del Instituto Mexicano del Seguro Social, 53(2), 240-249.

Figueroa, D. (2009). Obesidad y pobreza: marco conceptual para su análisis en Latinoamérica. Saúde e Sociedade Sao Paulo, 118(1), 103117.

Hersch-Martinez, P. (2013). Epidemiología sociocultural: una perspectiva necesaria. Salud pública de México, 512-518.

Honjo, K. (2004). Social Epidemiology: Definition, History and Research Examples. Environmental Health and Preventive Medicine, 9, 193-199.

Krieger, N. (2001). Historical Roots of Social Epidemiology: Socioeconomic Gradients in Health and Contextual Analysis. International Journal of Epidemiology, 30, 899-903.

Menéndez, E. L. (2008). Epidemiología sociocultural: propuestas y posibilidades. Región y sociedad, 2, 4-50.

Rodríguez-Torres, A. y Casas-Patiño, D. (2018). Determinantes sociales de la obesidad en México. Revista de enfermería del Instituto Mexicano del Seguro Social, 26(4), 281-290.

Suárez Lastra, M., Valdés González, C. y Galindo Pérez, M. C. (2020). Vulnerabilidad ante covid-19 en México. Visualizador de datos geográficos del iCOVID-19. Universidad Nacional Autónoma de México. URL: https://www.gits.igg.unam.mx/iCOVID-19/home.

Susser, M. (1987). Epidemiology in the U.S. After World War II: The Evolution of Technique. Epidemiologic Reviews, 7(1), 144-177. 
Susser, M. y Stein, Z. (2009). Eras in Epidemiology: The Evolution of Ideas. Oxford University Press.

Taylor, R. y Rieger, A. (1984). Rudolf Virchow and the Typhus Epidemic in Upper Silesia: An Introduction and Translation. Sociology of Health and Illness, 6, 201-217.

Van Rossum, C., Shipley, M., Van de Mheen, H., Grobbe, D. y Marmot, M. (2000). Employement Grade Differences in Cause Specific Mortality. a 25 Year Follow up of Civil Servants from the First Whitehall Study. Journal of Epidemiology and Community Health, 54, 178-184.

Villermé, L. R. (1976). Des épidemies sous les rapports de l'hygiène publique. En Annales d'hygiène publique et de médecine légale. Bibliothèque National de France. URL: https:/gallica.bnf.fr/ ark:/12148/bpt6k81426n.texteImage.

Vinten-Johansen, P., Brody, H., Paneth, N., Rachman, S. y Rip, M. (2003). Cholera, Chloroform, and the Science of Medicine: A Life of John Snow. Oxford University Press.

Rodrigo Itzamna Fuentes Reyes itzamnahjf@hotmail.com

Atocha Aliseda Universidad Nacional Autónoma de México. atocha@filosoficas.unam.mx 